\title{
Comparison Between Adomain Decomposition Method and Numerical Solutions of Linear Volterra Integral Equations of the Second Kind by Using the Fifth Order of Non-Polynomial Spline Functions
}

\author{
Elgaili Abdalla Elhassan Ibrahim, Abdel Radi Abdel Rahman Abdel Gadir Abdel Rahman², , \\ Neama Yahia Mohammed ${ }^{3}$, Nageeb Abdallah Hamed Haroun ${ }^{2}$ \\ ${ }^{1}$ Department of Mathematics, Faculty of Science, Sudan University for Science and Technology, Khartoum, Sudan \\ ${ }^{2}$ Department of Mathematics, Faculty of Education, Omdurman Islamic University, Omdurman, Sudan \\ ${ }^{3}$ Department of Mathematics, College of Science, Tabuk University, Tabuk, Saudi Arabia
}

Email address:

dibdelradi78@gmail.com (A. R. A. R. A. G. A. Rahman)

${ }^{*}$ Corresponding author

To cite this article:

Elgaili Abdalla Elhassan Ibrahim, Abdel Radi Abdel Rahman Abdel Gadir Abdel Rahman, Neama Yahia Mohammed, Nageeb Abdallah Hamed Haroun. Comparison Between Adomain Decomposition Method and Numerical Solutions of Linear Volterra Integral Equations of the Second Kind by Using the Fifth Order of Non-Polynomial Spline Functions. International Journal of Applied Mathematics and Theoretical Physics. Vol. 7, No. 3, 2021, pp. 68-79. doi: 10.11648/j.ijamtp.20210703.12

Received: August 11, 2021; Accepted: August 23, 2021; Published: August 31, 2021

\begin{abstract}
Volterra integral equations are a special type of integrative equations; they are divided into two categories referred to as the first and second type. This paper will deal with the second type which has wide range of the applications in physics and engineering problems. Spline functions are piece-wise polynomials of degree $n$ joined together at the break points with $n$-1 continuous derivatives. The break points of splines are called Knot, spline function can be integrated and differentiated due to being piece wise polynomials and can easily store and implemented on digital computer, non-polynomial spline function apiece wise is a blend of trigonometric, as well as, polynomial basis function, which form a complete extended Chebyshev space. Matlab is a powerful computing system for handling the calculations involved scientific and engineering problems. The aim of this paper is to compare between Adomain decomposition method and numerical solution to solve Volterra Integral Equations of second kind using the fifth order non-polynomial Spline functions by Matlab. We followed the applied mathematical method numerically by Matlab. Numerical examples are presented to illustrate the applications of this methods and to compare the computed results with analytical solutions. Finally by comparison of numerical results, Simplicity and efficiency of this method be shown.
\end{abstract}

Keywords: Linear Volterra Integral Equations, Second Kind, Non-Polynomial Spline Functions, Fifth Order, Adomain Decomposition Method

\section{Introduction}

Recently there is increase in concern by integrated equations, particularly Volterra integral equations has wide range of the applications in physics and engineering such as potential theory, Dirichlet problems, electrostatics, the particle transport problems of astrophysics, reactor problems, contact problems, diffusion problems and heat transfer problems(for more details see [4]).

In the mid of 1960s the numerical solution of integral equations was begun when the Kernel is unwell

In the beginning of 1980s the numerical methods began to take more importance when there is no analytical solution.

In 1997 Diogo, $\mathrm{T}$ and Lima, B presented deductive method [6], in order to find a numerical solution for Voltera integral equations with weakly singular kernel.

In 2007, Danaf \& Ramadan [11] presented applications of non-polynomial spline functions to solve Volterra equations.

In 2009 Haq [2] studied the numerical solution to the boundary value problem and the initial value problem using 
the Spline function.

In the year 2011, Zarebnia - Hoshyar - Sedahti [17] presented the numerical solution based on the Cube Spline function to find a solution to the boundary value problem.

In 2011 Majeed,S [8] presented a numerical solution to the Volterra differential integrative equation of the second kind using non-polynomial Spline functions.

In 2012 Hossinpour presented [5] a solution for differential integral equations by using non-polynomial spline functions.

In 2015, Harbis, S, Murad, M and Majed, S [3] presented a numerical solution for linear Voltera integral equation from second kind by using non-polynomial spline function from the third degree.

In the year 2016; Taqi. A, Jumma. B [15] Method of NonBoundary Spline Functions to Solve the System of Two Nonlinear Volterra Integral Equations.

In 2017, Najwa, S and Mohammed, S [7] presented a numerical solution for linear Voltera integral equations with weakly singular kernel by using non-polynomial spline function from the fifth degree.

In this paper we introduced a numerical solution for linear Voltera integral equation from second kind with by using nonpolynomial spline function from the fifth degree by Matlab.

\section{Analytical Solution of Linear Volterra Integral Equations of the Second Kind: $[1,9,16]$}

First we define Voltera integral equations of the second kind are given by:

$$
u(x)=f(x)+\int_{a}^{x} k(x, t) u(t) d t \quad 0 \leq x \leq b
$$

The unknown function $u(x)$ that determined, occurs inside and outside the integral sign, The Kernel $k(x, t)$ and the function $f(x)$ are given continues functions. $[1,9,16]$

There are many analytical methods available for solving Linear Volterra Integral Equations of second kind. We focus on the follow method: the Adomain decomposition method:

\section{Adomain Decomposition Method: $[1,9$, 16]}

The Adomain decomposition method (ADM) was introduced and developed by George Adomain. The Adomain decomposition method consists of decomposition the unknown function $u(x)$ of any equation into a sum of infinite number of components defined by the decompositions series

$$
u(x)=\sum_{n=0}^{n} u_{n}(x)
$$

Or equivalently

$$
u(x)=u_{0}(x)+u_{1}(x)+u_{2}(x)+\cdots
$$

Where the components $\left(u_{n}(x), n \geq 0\right.$ are to be determined in a recursive manner. The decomposition method concernsitself with finding the components individually; we substitute (2) into the Voltera integral equation (1) to obtain

$$
\sum_{n=0}^{\infty} u_{n}(x)=f(x)+\int_{a}^{x} k(x, t)\left(\sum_{n=0}^{\infty} u_{n}(t)\right) d t
$$

The zeroth components $u_{0}(x)$ is identified by all items that are not included under the integral sign. Consequently, the components $u_{j}(x), j \geq 1$ of the unknown function $u(x)$ is completely determined by setting the recurrence relation:

$$
\begin{gathered}
u_{0}(x)=f\left(x_{0}\right) \\
u_{n+1}(x)=\int_{a}^{x} k(x, t) u_{n}(t) d t, n \geq 0
\end{gathered}
$$

Example (1):[1,9,16]

To solve the following Voltera integral equation:

$$
u(x)=1-\int_{0}^{x} u(t) d t
$$

Where $f(x)=1$ and $k(x, t)=-1$,

Substituting decomposition series (2) into the both two sides of VIE's (12) gives

$$
\sum_{n=0}^{\infty} u_{n}(x)=1-\int_{0}^{x} \sum_{n=0}^{\infty} u_{n}(t) d t
$$

We identify the zeroth components by all terms that are not included under the integral sign. Therefore, we obtain the following recurrence relation:

$$
\begin{gathered}
u_{0}(x)=1, \\
u_{n+1}(x)=-\int_{0}^{x} u_{n}(t) d t, k \geq 0
\end{gathered}
$$

So that $u_{0}(x)=1$,

$$
\begin{gathered}
u_{1}(x)=-\int_{0}^{x} u_{0}(t) d t=-\int_{0}^{x} 1 d t=-x, \\
u_{2}(x)=-\int_{0}^{x} u_{1}(t) d t=-\int_{0}^{x}-t d t=\frac{x^{2}}{2 !}, \\
u_{3}(x)=-\int_{0}^{x} u_{2}(t) d t=-\int_{0}^{x} \frac{t^{2}}{2} d t=-\frac{x^{3}}{3 !}, \\
u_{4}(x)=-\int_{0}^{x} u_{3}(t) d t=-\int_{0}^{x}-\frac{t^{3}}{6} d t,=\frac{x^{4}}{4 !}, \forall \text { equations }
\end{gathered}
$$

And so on, gives the series solution

$$
u(x)=1-x+\frac{x^{2}}{2 !}+\frac{x^{3}}{3 !}+\cdots=e^{-x}
$$

which is equation (11) the exact solution for equation (7)

\section{A Numerical Solution of Linear VIE's of 2 nd Kind Using the 5th Order of Non-Polynomial Spline Functions}

The form of the five non-polynomial spline function is: 


$$
P_{i}(t)=a_{i} \cos K\left(t-t_{i}\right)+b_{i} \sin K\left(t-t_{i}\right)+c_{i}\left(t-t_{i}\right)+d_{i}\left(t-t_{i}\right)^{2}+e_{i}\left(t-t_{i}\right)^{3}+r_{i}\left(t-t_{i}\right)^{4}+z_{i}\left(t-t_{i}\right)^{5}+g_{i}
$$

where $a_{i}, b_{i}, c_{i}, d_{i}, e_{i}, r_{i}, z_{i}$, and $g_{i}$ are constants, to be determined. In order to obtain the values of $a_{i}, b_{i}, c_{i}, d_{i}, e_{i}, r_{i}, z_{i}$, and $g_{i}$, we differentiate equation (12) seven times with respect to $t$, and we get the following equations

$$
\begin{aligned}
& P_{i}^{(1)}(t)=-K a_{i} \sin K\left(t-t_{i}\right)+K b_{i} \cos K\left(t-t_{i}\right)+c_{i}+2 d_{i}\left(t-t_{i}\right)+3 e_{i}\left(t-t_{i}\right)^{2} \\
& +4 r_{i}\left(t-t_{i}\right)^{3}+5 z_{i}\left(t-t_{i}\right)^{4} \\
& P_{i}^{(2)}(t)=-K^{2} a_{i} \cos K\left(t-t_{i}\right)-K^{2} b_{i} \sin K\left(t-t_{i}\right)+2 d_{i}+6 e_{i}\left(t-t_{i}\right) \\
& +12 r_{i}\left(t-t_{i}\right)^{2}+20 z_{i}\left(t-t_{i}\right)^{3} \\
& \left.P_{i}^{(3)}(t)=K^{3} a_{i} \sin K\left(t-t_{i}\right)-K^{3} b_{i} \cos K\left(t-t_{i}\right)+6 e_{i}+24 r_{i}\left(t-t_{i}\right)+60 z_{i}\left(t-t_{i}\right)^{2}\right\} \\
& P_{i}^{(4)}(t)=K^{4} a_{i} \cos K\left(t-t_{i}\right)+K^{4} b_{i} \sin K\left(t-t_{i}\right)+24 r_{i}+120 z_{i}\left(t-t_{i}\right) \\
& P_{i}^{(5)}(t)=-K^{5} a_{i} \sin K\left(t-t_{i}\right)+K^{5} b_{i} \cos K\left(t-t_{i}\right)+120 z_{i} \\
& P_{i}^{(6)}(t)=-K^{6} a_{i} \cos K\left(t-t_{i}\right)-K^{6} b_{i} \sin K\left(t-t_{i}\right) \\
& P_{i}^{(7)}(t)=K^{7} a_{i} \sin K\left(t-t_{i}\right)-K^{7} b_{i} \cos K\left(t-t_{i}\right)
\end{aligned}
$$

Hence replace $t$ by $t_{i}$ in the relation (12) and (13) yields:

$$
\begin{gathered}
P(t)=a_{i}+g_{i} \\
P_{i}^{(1)}(t)=b_{i} K+c_{i} \\
P_{i}^{(2)}(t)=-K^{2} a_{i}+2 d_{i} \\
P_{i}^{(3)}(t)=-K^{3} b_{i}+6 e_{i} \\
P_{i}^{(4)}(t)=K^{4} a_{i}+24 r_{i} \\
P_{i}^{(5)}(t)=K^{5} a_{i}+120 z_{i} \\
P_{i}^{(6)}(t)=-K^{6} a_{i} \\
P_{i}^{(7)}(t)=-K^{7} b_{i}
\end{gathered}
$$

We obtained the values of $a_{i}, b_{i}, c_{i}, d_{i}, e_{i}, r_{i}, z_{i}$, and $g_{i}$ from the above relations as follows:

$$
\begin{aligned}
& a_{i}=-\frac{1}{K^{6}} P_{i}^{(6)}(t) \\
& b_{i}=\frac{1}{K^{7}} P_{i}^{(7)}(t) \\
& c_{i}=P_{i}^{(1)}(t)-K b_{i} \\
& u^{(2)}(x)=f^{(2)}(x)+\int_{a}^{x} \frac{\partial^{(2)} k(x, t)}{\partial x^{(2)}} u(t) d t+\left(\frac{\partial k(x, t)}{\partial x}\right)_{t=x} u(x)+\frac{d k(x, x)}{d x} u(x)+k(x, x) u^{(1)}(x) \\
& u^{(3)}(x)=f^{(3)}(x)+\int_{a}^{x} \frac{\partial^{(3)} k(x, t)}{\partial x^{(3)}} u(t) d t+\left(\frac{\partial^{(2)} k(x, t)}{\partial x^{(2)}}\right)_{t=x} u(x)+\frac{d}{d x}\left(\frac{\partial k(x, t)}{\partial x}\right)_{t=x} u(x)+\left(\frac{\partial k(x, t)}{\partial x}\right)_{t=x} u^{(1)}(x)+\frac{d^{(2)} k(x, x)}{d x^{(2)}} u(x)+ \\
& 2 \frac{d k(x, x)}{d x} u^{(1)}(x)+k(x, x) u^{(2)}(x) \\
& u^{(4)}(x)=f^{(4)}(x)+\int_{a}^{x} \frac{\partial^{(4)} k(x, t)}{\partial x^{(4)}} u(t) d t+\left(\frac{\partial^{(3)} k(x, t)}{\partial x^{(3)}}\right)_{t=x} u(x)+\frac{d}{d x}\left(\frac{\partial^{(2)} k(x, t)}{\partial x^{(2)}}\right)_{t=x} u(x)+\left(\frac{\partial^{(2)} k(x, t)}{\partial x^{(2)}}\right)_{t=x} u^{(1)}(x)+ \\
& \frac{d^{(2)}}{d x^{(2)}}\left(\frac{\partial k(x, t)}{\partial x}\right)_{t=x} u(x)+2 \frac{d}{d x}\left(\frac{\partial k(x, t)}{\partial x}\right)_{t=x} u^{(1)}(x)+\left(\frac{\partial k(x, t)}{\partial x}\right)_{t=x} u^{(2)}(x)+\frac{d^{(3)} k(x, x)}{d x^{(3)}} u(x)+3 \frac{d^{(2)} k(x, x)}{d x^{(2)}} u^{(1)}(x)+ \\
& 3 \frac{d k(x, x)}{d x} u^{(2)}(x)+k(x, x) u^{(3)}(x) \\
& u(x)=f(x)+\int_{a}^{x} k(x, t) u(t) d t 0 \leq x \leq b \\
& u^{(1)}(x)=f^{(1)}(x)+\int_{a}^{x} \frac{\partial k(x, t)}{\partial x} u(t) d t+k(x, x) u(x)
\end{aligned}
$$

$$
\begin{array}{r}
d_{i}=\frac{1}{2}\left[P_{i}^{(2)}(t)+K^{2} a_{i}\right] \\
e_{i}=\frac{1}{6}\left[P_{i}^{(3)}(t)+K^{3} b_{i}\right] \\
r_{i}=\frac{1}{24}\left[P_{i}^{(4)}(t)-K^{4} a_{i}\right] \\
z_{i}=\frac{1}{120}\left[P_{i}^{(5)}(t)-K^{5} b_{i}\right] \\
g_{i}=P_{i}(t)-a_{i}
\end{array}
$$

for $i=0,1, \ldots, n$

\section{Linear Volterra Integral Equations of the Second Kind [1, 9, 16]}

First we define Volterra integral equations of the second kind are given by: 


$$
\begin{aligned}
& u^{(5)}(x)=f^{(5)}(x)+\int_{a}^{x} \frac{\partial^{(5)} k(x, t)}{\partial x^{(5)}} u(t) d t+\left(\frac{\partial^{(4)} k(x, t)}{\partial x^{(4)}}\right)_{t=x} u(x)+\frac{d}{d x}\left(\frac{\partial^{(3)} k(x, t)}{\partial x^{(3)}}\right)_{t=x} u(x)+\left(\frac{\partial^{(3)} k(x, t)}{\partial x^{(3)}}\right)_{t=x} u^{(1)}(x)+ \\
& \frac{d^{(2)}}{d x^{(2)}}\left(\frac{\partial^{(2)} k(x, t)}{\partial x^{(2)}}\right)_{t=x} u(x)+2 \frac{d}{d x}\left(\frac{\partial^{(2)} k(x, t)}{\partial x^{(2)}}\right)_{t=x} u^{(1)}(x)+\left(\frac{\partial^{(2)} k(x, t)}{\partial x^{(2)}}\right)_{t=x} u^{(2)}(x)+\frac{d^{(3)}}{d x^{(3)}}\left(\frac{\partial k(x, t)}{\partial x}\right)_{t=x} u(x)+ \\
& 3 \frac{d^{(2)}}{d x^{(2)}}\left(\frac{\partial k(x, t)}{\partial x}\right)_{t=x} u^{(1)}(x)+3 \frac{d}{d x}\left(\frac{\partial k(x, t)}{\partial x}\right)_{t=x} u^{(2)}(x)+\left(\frac{\partial k(x, t)}{\partial x}\right)_{t=x} u^{(3)}(x)+\frac{d^{(4)} k(x, x)}{d x^{(4)}} u(x)+4 \frac{d^{(3)} k(x, x)}{d x^{(3)}} u^{(1)}(x)+ \\
& 6 \frac{d^{(2)} k(x, x)}{d x^{(2)}} u^{(2)}(x)+4 \frac{d k(x, x)}{d x} u^{(3)}(x)+k(x, x) u^{(4)}(x) \\
& u^{(6)}(x)=f^{(6)}(x)+\int_{a}^{x} \frac{\partial^{(6)} k(x, t)}{\partial x^{(6)}} u(t) d t+\left(\frac{\partial^{(5)} k(x, t)}{\partial x^{(5)}}\right)_{t=x} u(x)+\frac{d}{d x}\left(\frac{\partial^{(4)} k(x, t)}{\partial x^{(4)}}\right)_{t=x} u(x)+\left(\frac{\partial^{(4)} k(x, t)}{\partial x^{(4)}}\right)_{t=x} u^{(1)}(x)+ \\
& \frac{d}{d x}\left(\frac{\partial^{(3)} k(x, t)}{\partial x^{(3)}}\right)_{t=x} u^{1}(x)+2 \frac{d}{d x}\left(\frac{\partial^{(3)} k(x, t)}{\partial x^{(3)}}\right)_{t=x} u^{(1)}(x)+\left(\frac{\partial^{(3)} k(x, t)}{\partial x^{(3)}}\right)_{t=x} u^{(2)}(x)+\frac{d^{(3)}}{d x^{(3)}}\left(\frac{\partial^{(2)} k(x, t)}{\partial x^{(2)}}\right)_{t=x} u(x)+ \\
& 3 \frac{d^{(2)}}{d x^{(2)}}\left(\frac{\partial^{(2)} k(x, t)}{\partial x^{(2)}}\right)_{t=x} u^{(1)}(x)+3 \frac{d}{d x}\left(\frac{\partial^{(2)} k(x, t)}{\partial x^{(2)}}\right)_{t=x} u^{(2)}(x)+\left(\frac{\partial^{(2)} k(x, t)}{\partial x^{(2)}}\right)_{t=x} u^{(3)}(x)+\frac{d^{(4)}}{d x^{(4)}}\left(\frac{\partial k(x, t)}{\partial x}\right)_{t=x} u(x)+ \\
& 4 \frac{d^{(3)}}{d x^{(3)}}\left(\frac{\partial k(x, t)}{\partial x}\right)_{t=x} u^{(1)}(x)+6 \frac{d^{(2)}}{d x^{(2)}}\left(\frac{\partial k(x, t)}{\partial x}\right)_{t=x} u^{(2)}(x)+4 \frac{d}{d x}\left(\frac{\partial k(x, t)}{\partial x}\right)_{t=x} u^{(3)}(x)+\left(\frac{\partial k(x, t)}{\partial x}\right)_{t=x} u^{(4)}(x)+\frac{d^{5} k(x, x)}{d x^{(5)}} u(x)+ \\
& 5 \frac{d^{(4)} k(x, x)}{d x^{(4)}} u^{(1)}(x)+10 \frac{d^{(3)} k(x, x)}{d x^{(3)}} u^{(2)}(x)+10 \frac{d^{(2)} k(x, x)}{d x^{(2)}} u^{(3)}(x)+5 \frac{d k(x, x)}{d x} u^{(4)}(x)+k(x, x) u^{(5)}(x) \\
& u^{(7)}(x)=f^{(7)}(x)+\int_{a}^{x} \frac{\partial^{(7)} k(x, t)}{\partial x^{(7)}} u(t) d t+\left(\frac{\partial^{(6)} k(x, t)}{\partial x^{(6)}}\right)_{t=x} u(x)+\frac{d}{d x}\left(\frac{\partial^{(5)} k(x, t)}{\partial x^{(5)}}\right)_{t=x} u(x)+\left(\frac{\partial^{(5)} k(x, t)}{\partial x^{(5)}}\right)_{t=x} u^{(1)}(x)+ \\
& \frac{d^{2}}{d x^{2}}\left(\frac{\partial^{(4)} k(x, t)}{\partial x^{(4)}}\right)_{t=x} u(x)+2 \frac{d}{d x}\left(\frac{\partial^{(4)} k(x, t)}{\partial x^{(4)}}\right)_{t=x} u^{(1)}(x)+\left(\frac{\partial^{(4)} k(x, t)}{\partial x^{(4)}}\right)_{t=x} u^{(2)}(x)+\frac{d^{(3)}}{d x^{(3)}}\left(\frac{\partial^{(3)} k(x, t)}{\partial x^{(3)}}\right)_{t=x} u(x)+ \\
& 3 \frac{d^{(2)}}{d x^{(2)}}\left(\frac{\partial^{(3)} k(x, t)}{\partial x^{(3)}}\right)_{t=x} u^{(1)}(x)+3 \frac{d}{d x}\left(\frac{\partial^{(3)} k(x, t)}{\partial x^{(3)}}\right)_{t=x} u^{(2)}(x)+\frac{d^{(4)}}{d x^{(4)}}\left(\frac{\partial^{(2)} k(x, t)}{\partial x^{(2)}}\right)_{t=x} u(x)+4 \frac{d^{(3)}}{d x^{(3)}}\left(\frac{\partial^{(2)} k(x, t)}{\partial x^{(2)}}\right)_{t=x} u^{(1)}(x)+ \\
& 6 \frac{d^{(2)}}{d x^{(2)}}\left(\frac{\partial^{(2)} k(x, t)}{\partial x^{(2)}}\right)_{t=x} u^{(2)}(x)+4 \frac{d}{d x}\left(\frac{\partial^{(2)} k(x, t)}{\partial x^{(2)}}\right)_{t=x} u^{(3)}(x)+\left(\frac{\partial^{(3)} k(x, t)}{\partial x^{(3)}}\right)_{t=x} u^{(3)}(x)+\left(\frac{\partial^{(2)} k(x, t)}{\partial x^{(2)}}\right)_{t=x} u^{(4)}(x)+ \\
& \left(\frac{d^{(5)}}{d x^{(5)}}\left(\frac{\partial k(x, t)}{\partial x}\right)\right) u(x)+5 \frac{d^{(4)}}{d x^{(4)}}\left(\frac{\partial k(x, t)}{\partial x}\right)_{t=x} u^{(1)}(x)+10 \frac{d^{(3)}}{d x^{(3)}}\left(\frac{\partial k(x, t)}{\partial x}\right)_{t=x} u^{(2)}(x)+10 \frac{d^{(2)}}{d x^{(2)}}\left(\frac{\partial k(x, t)}{\partial x}\right)_{t=x} u^{(3)}(x)+ \\
& 5 \frac{d}{d x}\left(\frac{\partial k(x, t)}{\partial x}\right)_{t=x} u^{(4)}(x)+\left(\frac{\partial k(x, t)}{\partial x}\right)_{t=x} u^{(5)}(x)+\frac{d^{(6)} k(x, x)}{d x^{(6)}} u(x)+6 \frac{d^{(5)} k(x, x)}{d x^{(5)}} u^{(1)}(x)+15 \frac{d^{(4)} k(x, x)}{d x^{(4)}} u^{(2)}(x)+ \\
& 20 \frac{d^{(3)} k(x, x)}{d x^{(3)}} u^{(3)}(x)+15 \frac{d^{(2)} k(x, x)}{d x^{(2)}} u^{(4)}(x)+6 \frac{d k(x, x)}{d x} u^{(5)}(x)+k(x, x) u^{(6)}(x)
\end{aligned}
$$

To complete our procedure for solving VIE's we substitute $x=a$ in equations (22)-(29) then we get:

$$
\begin{aligned}
& u_{0}=u(a)=f(a) \\
& u_{0}^{(1)}=u^{(1)}(a)=f^{(1)}(a)+k(a, a) \cdot u(a) \\
& u_{0}^{(2)}=u^{(2)}(a)=f^{(2)}(a)+\left(\left(\frac{\partial k(x, t)}{\partial x}\right)_{t=x}\right)_{x=a} u(a)+\left(\frac{d k(x, x)}{d x}\right)_{x=a} u(a)+k(a, a) u^{(1)}(a) \\
& u_{0}^{(3)}=u^{(3)}(a)=f^{(3)}(a)+\left(\left(\frac{\partial^{(2)} k(x, t)}{\partial x^{(2)}}\right)_{t=x}\right)_{x=a} u(a)+\left(\frac{d}{d x}\left(\frac{\partial k(x, t)}{\partial x}\right)_{t=x}\right)_{x=a} u(a)+\left(\left(\frac{\partial k(x, t)}{\partial x}\right)_{t=x}\right)_{x=a} u^{(1)}(a)+ \\
& \left(\frac{d^{(2)} k(x, x)}{d x^{(2)}}\right)_{x=a} u(a)+2\left(\frac{d k(x, x)}{d x}\right)_{x=a} u^{(1)}(a)+k(x, x) u^{(2)}(a) \\
& u_{0}^{(4)}=u^{(4)}(a)=f^{(4)}(a)+\left(\left(\frac{\partial^{(3)} k(x, t)}{\partial x^{(3)}}\right)_{t=x}\right)_{x=a} u(a)+\left(\frac{d}{d x}\left(\frac{\partial^{(2)} k(x, t)}{\partial x^{(2)}}\right)_{t=x}\right)_{x=a} u(a)+\left(\left(\frac{\partial^{(2)} k(x, t)}{\partial x^{(2)}}\right)_{t=x}\right)_{x=a} u^{(1)}(a)+ \\
& \left(\frac{d^{(2)}}{d x^{(2)}}\left(\frac{\partial k(x, t)}{\partial x}\right)_{t=x}\right)_{x=a} u(a)+2\left(\frac{d}{d x}\left(\frac{\partial k(x, t)}{\partial x}\right)_{t=x}\right)_{x=a} u^{(1)}(a)+\left(\left(\frac{\partial k(x, t)}{\partial x}\right)_{t=x}\right)_{x=a} u^{(2)}(a)+\left(\frac{d^{(3)} k(x, x)}{d x^{(3)}}\right)_{x=a} u(a)+ \\
& 3\left(\frac{d^{(2)} k(x, x)}{d x^{(2)}}\right)_{x=a} u^{(1)}(a)+3\left(\frac{d k(x, x)}{d x}\right)_{x=a} u^{(2)}(a)+k(x, x) u^{(3)}(a) \\
& u_{0}^{(5)}=u^{(5)}(a)=f^{(5)}(a)+\left(\left(\frac{\partial^{(4)} k(x, t)}{\partial x^{(4)}}\right)_{t=x}\right)_{x=a} u(a)+\left(\frac{d}{d x}\left(\frac{\partial^{(3)} k(x, t)}{\partial x^{(3)}}\right)_{t=x}\right)_{x=a} u(a)+\left(\left(\frac{\partial^{(3)} k(x, t)}{\partial x^{(3)}}\right)_{t=x}\right)_{x=a} u^{(1)}(a)+ \\
& \left(\frac{d^{(2)}}{d x^{(2)}}\left(\frac{\partial^{(2)} k(x, t)}{\partial x^{(2)}}\right)_{t=x}\right)_{x=a} u(a)+2\left(\frac{d}{d x}\left(\frac{\partial^{(2)} k(x, t)}{\partial x^{(2)}}\right)_{t=x}\right)_{x=a} u^{(1)}(a)+\left(\left(\frac{\partial^{(2)} k(x, t)}{\partial x^{(2)}}\right)_{t=x}\right)_{x=a} u^{(2)}(a)+ \\
& \left(\frac{d^{(3)}}{d x^{(3)}}\left(\frac{\partial k(x, t)}{\partial x}\right)_{t=x}\right)_{x=a} u(a)+3\left(\frac{d^{(2)}}{d x^{(2)}}\left(\frac{\partial k(x, t)}{\partial x}\right)_{t=x}\right)_{x=a} u^{(1)}(a)+3\left(\frac{d}{d x}\left(\frac{\partial k(x, t)}{\partial x}\right)_{t=x}\right)_{x=a} u^{(2)}(a)+ \\
& \left(\left(\frac{\partial k(x, t)}{\partial x}\right)_{t=x}\right)_{x=a} u^{(3)}(a)+\left(\frac{d^{(4)} k(x, x)}{d x^{(4)}}\right)_{x=a} u(a)+4\left(\frac{d^{(3)} k(x, x)}{d x^{(3)}}\right)_{x=a} u^{(1)}(a)+6\left(\frac{d^{(2)} k(x, x)}{d x^{(2)}}\right)_{x=a} u^{(2)}(a)+
\end{aligned}
$$




$$
\begin{aligned}
& 4\left(\frac{d k(x, x)}{d x}\right)_{x=a} u^{(3)}(a)+k(x, x) u^{(4)}(a) \\
& u_{0}^{(6)}=u^{(6)}(a)=f^{(6)}(a)+\left(\left(\frac{\partial^{(5)} k(x, t)}{\partial x^{(5)}}\right)_{t=x}\right)_{x=a} u(a)+\left(\frac{d}{d x}\left(\frac{\partial^{(4)} k(x, t)}{\partial x^{(4)}}\right)_{t=x}\right)_{x=a} u(a)+\left(\left(\frac{\partial^{(4)} k(x, t)}{\partial x^{(4)}}\right)_{t=x}\right)_{x=a} u^{(1)}(a)+ \\
& \left(\frac{d^{(2)}}{d x^{(2)}}\left(\frac{\partial^{(3)} k(x, t)}{\partial x^{(3)}}\right)_{t=x}\right)_{x=a} u(a)+2\left(\frac{d}{d x}\left(\frac{\partial^{(3)} k(x, t)}{\partial x^{(3)}}\right)_{t=x}\right)_{x=a} u^{(1)}(a)+\left(\left(\frac{\partial^{(3)} k(x, t)}{\partial x^{(3)}}\right)_{t=x}\right)_{x=a} u^{(2)}(a)+ \\
& \left(\frac{d^{(3)}}{d x^{(3)}}\left(\frac{\partial^{(2)} k(x, t)}{\partial x^{(2)}}\right)_{t=x}\right)_{x=a} u(a)+3\left(\frac{d^{(2)}}{d x^{(2)}}\left(\frac{\partial^{(2)} k(x, t)}{\partial x^{(2)}}\right)_{t=x}\right)_{x=a} u^{(1)}(a)+3\left(\frac{d}{d x}\left(\frac{\partial^{(2)} k(x, t)}{\partial x^{(2)}}\right)_{t=x}\right)_{x=a} u^{(2)}(a)+ \\
& \left(\left(\frac{\partial^{(2)} k(x, t)}{\partial x^{(2)}}\right)_{t=x}\right)_{x=a} u^{(3)}(a)+\left(\frac{d^{(4)}}{d x^{(4)}}\left(\frac{\partial k(x, t)}{\partial x}\right)_{t=x}\right)_{x=a} u(a)+4\left(\frac{d^{(3)}}{d x^{(3)}}\left(\frac{\partial k(x, t)}{\partial x}\right)_{t=x}\right)_{x=a}^{x=a} u^{(1)}(a)+ \\
& 6\left(\frac{d^{(2)}}{d x^{(2)}}\left(\frac{\partial k(x, t)}{\partial x}\right)_{t=x}\right)_{x=a} u^{(2)}(x)+4\left(\frac{d}{d x}\left(\frac{\partial k(x, t)}{\partial x}\right)_{t=x}\right)_{x=a} u^{(3)}(a)+\left(\left(\frac{\partial k(x, t)}{\partial x}\right)_{t=x}\right)_{x=a} u^{(4)}(a)+\left(\frac{d^{(5)} k(x, x)}{d x^{(5)}}\right)_{x=a} u(a)+ \\
& 5\left(\frac{d^{(4)} k(x, x)}{d x^{(4)}}\right)_{x=a} u^{(1)}(a)+10\left(\frac{d^{(3)} k(x, x)}{d x^{(3)}}\right)_{x=a} u^{(2)}(a)+10\left(\frac{d^{(2)} k(x, x)}{d x^{(2)}}\right)_{x=a} u^{(3)}(a)+5\left(\frac{d k(x, x)}{d x}\right)_{x=a} u^{(4)}(a)+k(x, x) u^{(5)}(a) \\
& u_{0}^{(7)}=u^{(7)}(a)=f^{(7)}(a)=\left(\left(\frac{\partial^{(6)} k(x, t)}{\partial x^{(6)}}\right)_{t=x}\right)_{x=a} u(a)+\left(\frac{d}{d x}\left(\frac{\partial^{(5)} k(x, t)}{\partial x^{(5)}}\right)_{t=x}\right)_{x=a} u(a)+\left(\left(\frac{\partial^{(5)} k(x, t)}{\partial x^{(5)}}\right)_{t=x}\right)_{x=a} u^{(1)}(a)+ \\
& \left(\frac{d^{(2)}}{d x^{(2)}}\left(\frac{\partial^{(4)} k(x, t)}{\partial x^{(4)}}\right)_{t=x}\right)_{x=a} u(a)+2\left(\frac{d}{d x}\left(\frac{\partial^{(4)} k(x, t)}{\partial x^{(4)}}\right)_{t=x}\right)_{x=a} u^{(1)}(a)+\left(\left(\frac{\partial^{(4)} k(x, t)}{\partial x^{(4)}}\right)_{t=x}\right)_{x=a} u^{(2)}(a)+ \\
& \left(\frac{d^{(3)}}{d x^{(3)}}\left(\frac{\partial^{(3)} k(x, t)}{\partial x^{(3)}}\right)_{t=x}\right)_{x=a} u(a)+3\left(\frac{d^{(2)}}{d x^{(2)}}\left(\frac{\partial^{(3)} k(x, t)}{\partial x^{(3)}}\right)_{t=x}\right)_{x=a} u^{(1)}(a)+3\left(\frac{d}{d x}\left(\frac{\partial^{(3)} k(x, t)}{\partial x^{(3)}}\right)_{t=x}\right)_{x=a}^{x} u^{(2)}(a)+ \\
& \left(\frac{d^{(4)}}{d x^{(4)}}\left(\frac{\partial^{(2)} k(x, t)}{\partial x^{(2)}}\right)_{t=x}\right)_{x=a} u(a)+4\left(\frac{d^{(3)}}{d x^{(3)}}\left(\frac{\partial^{(2)} k(x, t)}{\partial x^{(2)}}\right)_{t=x}\right)_{x=a} u^{(1)}(a)+6\left(\frac{d^{(2)}}{d x^{(2)}}\left(\frac{\partial^{(2)} k(x, t)}{\partial x^{(2)}}\right)_{t=x}\right)_{x=a} u^{(2)}(a)+ \\
& 4\left(\frac{d}{d x}\left(\frac{\partial^{(2)} k(x, t)}{\partial x^{(2)}}\right)_{t=x}\right)_{x=a} u^{(3)}(a)+\left(\left(\frac{\partial^{(3)} k(x, t)}{\partial x^{(3)}}\right)_{t=x}\right)_{x=a} u^{(3)}(a)+\left(\left(\frac{\partial^{(2)} k(x, t)}{\partial x^{(2)}}\right)_{t=x}\right)_{x=a} u^{(4)}(a)+ \\
& \left(\frac{d^{(5)}}{d x^{(5)}}\left(\frac{\partial k(x, t)}{\partial x}\right)_{t=x}\right)_{x=a} u(a)+5\left(\frac{d^{(4)}}{d x^{(4)}}\left(\frac{\partial k(x, t)}{\partial x}\right)_{t=x}\right)_{x=a} u^{(1)}(a)+10\left(\frac{d^{(3)}}{d x^{(3)}}\left(\frac{\partial k(x, t)}{\partial x}\right)_{t=x}\right)_{x=a} u^{(2)}(a)+ \\
& 10\left(\frac{d^{(2)}}{d x^{(2)}}\left(\frac{\partial k(x, t)}{\partial x}\right)_{t=x}\right)_{x=a} u^{(3)}(a)+5\left(\frac{d}{d x}\left(\frac{\partial k(x, t)}{\partial x}\right)_{t=x}\right)_{x=a} u^{(4)}(a)+\left(\left(\frac{\partial k(x, t)}{\partial x}\right)_{t=x}\right)_{x=a} u^{(5)}(a)+\left(\frac{d^{(6)} k(x, x)}{d x^{(6)}}\right)_{x=a} u(a)+ \\
& 6\left(\frac{d^{(5)} k(x, x)}{d x^{(5)}}\right)_{x=a} u^{(1)}(a)+15\left(\frac{d^{(4)} k(x, x)}{d x^{(4)}}\right)_{x=a} u^{(2)}(a)+20\left(\frac{d^{(3)} k(x, x)}{d x^{(3)}}\right)_{x=a} u^{(3)}(a)+15\left(\frac{d^{(2)} k(x, x)}{d x^{(2)}}\right)_{x=a} u^{(4)}(a)+ \\
& 6\left(\frac{d k(x, x)}{d x}\right)_{x=a} u^{(5)}(a)+k(x, x) u^{(6)}(a)
\end{aligned}
$$

Now, we try to solve equation (22) using fifth order nonpolynomial Spline functions.

\section{Using Fifth Order Non-Polynomial Spline Function}

In order to approximate the solution of linear VIE's of second kind (22) by using fifth order non-polynomial spline function (1). we present a method of solution in algorithm (VIE2NPS5).

The algorithm (VIE2NPS5):

Step 1: set $h=\frac{b-a}{n}, t_{i}=t_{0}+i h, i=0,1, \ldots, n$

where $t_{0}=a, t_{n}=b$ and $u_{0}=f(a)$.

Step 2: Evaluate $a_{0}, b_{0}, c_{0}, d_{0}, e_{0}, r_{0} z_{0}$ and $g_{0}$ by substituting (30)-(37) In equations (13) - (21).

Step 3: Calculate $p_{0}(t)$ using step 2 and equation (12).

Step 4: Approximate $u_{1}=p_{0}\left(t_{i}\right)$.

Step 5: For $i=1$ to $n-1$ do following steps:

Step 6: Evaluate $a_{i}, b_{i}, c_{i}, d_{i}, e_{i}, r_{i}, z_{i}$ and $g_{i}$ by using equations (13)-(21) and replacing $u\left(t_{i}\right), u^{1}\left(t_{i}\right), u^{2}\left(t_{i}\right), u^{3}\left(t_{i}\right), u^{4}\left(t_{i}\right), u^{5}\left(t_{i}\right), u^{6}\left(t_{i}\right)$ and $u^{7}\left(t_{i}\right)$

by $p_{i}\left(t_{i}\right), p_{i}{ }^{1}\left(t_{i}\right), p_{i}{ }^{2}\left(t_{i}\right), p_{i}{ }^{3}\left(t_{i}\right), p_{i}{ }^{4}\left(t_{i}\right), p_{i}{ }^{5}\left(t_{i}\right), p_{i}{ }^{6}\left(t_{i}\right)$ and $p_{i}{ }^{7}\left(t_{i}\right)$

Step 7: Calculate $p_{i}(t)$ using step 6 and equation (12).
Step 8: Approximate $u_{i+1}=p_{i}\left(t_{i+1}\right)$.

\section{Programming}

Program 1: linear non-polynomial spline function for solving VIE's of the second kind.

function $[\mathrm{x}, \mathrm{y}, \mathrm{err}, \mathrm{u}]=$ volnonpolyspline $1 \mathrm{st}(\mathrm{ker}, \mathrm{f}, \mathrm{ex}, \mathrm{a}, \mathrm{b}, \mathrm{n})$ syms X T s

$\mathrm{h}=(\mathrm{b}-\mathrm{a}) /(\mathrm{n}-1)$;

$\mathrm{u}=\operatorname{subs}(\mathrm{f}, \mathrm{X}, \mathrm{a})$

ifisempty $(\operatorname{diff}(f, 1))==1$

$\mathrm{z} 1=0$;

else

$\mathrm{z} 1=\operatorname{diff}(\mathrm{f}, 1)$

end

$\mathrm{du}=\operatorname{subs}(\mathrm{z} 1, \mathrm{a})+\operatorname{subs}(\mathrm{ker},\{\mathrm{X}, \mathrm{T}\},\{\mathrm{a}, \mathrm{a}\})^{*} \mathrm{u}(1)$;

ifisempty $(\operatorname{diff}(f, 2))==1$

$\mathrm{z} 2=0$;

else

$\mathrm{z} 2=\operatorname{diff}(\mathrm{f}, 2)$

end

ifisempty $\left(\operatorname{diff}\left(\operatorname{subs}\left(\operatorname{ker},\{\mathrm{T}\},\{\mathrm{X}\}, \mathrm{X}^{\prime}\right)\right)\right)==1$

$\mathrm{z} 3=0$;

else 


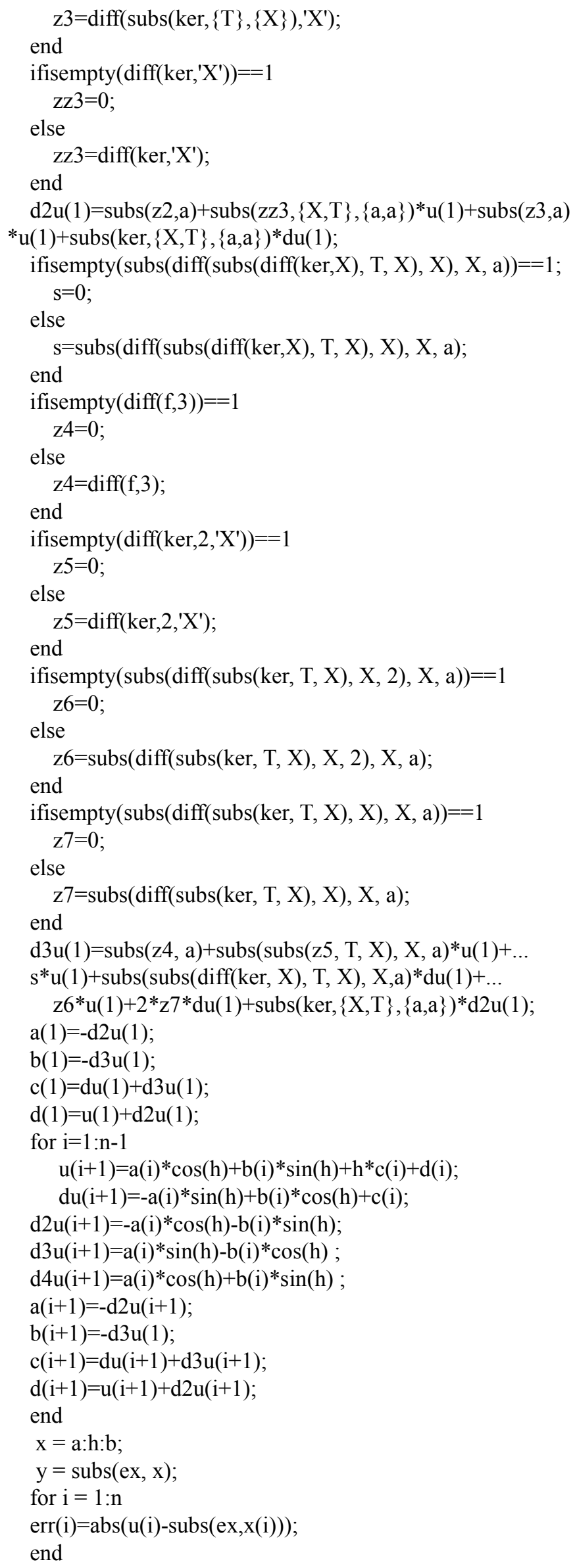

\section{format long}

$\operatorname{disp}()^{\prime} i$

[1:n; $x ; y ; u ; e r r]^{\prime}$

figure(1)

plot(x, y, 'b --', 'linewidth', 3)

hold on

plot(x, u, 'r.-', 'linewidth', 1)

grid on

xlabel('X')

ylabel('Y')

title('exact vs. approx.')

legend('exact', 'approx.')

Program 2: sixth no -polynomial spline function for solving VIE's of the second kind with weakly singular kernel. function $[X, Y$, err, u] = volnonpolyspline5st (ker,f, ex, a, b, n) syms X T

$\mathrm{h}=(\mathrm{b}-\mathrm{a}) /(\mathrm{n}-1)$;

$\mathrm{u}=\operatorname{subs}(\mathrm{f}, \mathrm{X}, \mathrm{a})$

$\mathrm{x}=\mathrm{a}: \mathrm{h}: \mathrm{b}$;

$y=\operatorname{subs}(e x, x)$;

ifisempty $(\operatorname{diff}(f, 1))==1$

$$
\mathrm{z} 1=0 \text {; }
$$

else

$$
\mathrm{z} 1=\operatorname{diff}(\mathrm{f}, 1)
$$

end

$\mathrm{du}=\operatorname{subs}(\mathrm{z} 1, \mathrm{a})+\operatorname{subs}(\operatorname{ker},\{\mathrm{X}, \mathrm{T}\},\{\mathrm{a}, \mathrm{a}\})^{*} \mathrm{u}(1)$;

ifisempty $(\operatorname{diff}(\mathrm{f}, 2))==1$

$$
\mathrm{z} 2=0 \text {; }
$$

else

$$
z 2=\operatorname{diff}(f, 2) \text {; }
$$

end

ifisempty $\left(\operatorname{diff}\left(\operatorname{subs}\left(\operatorname{ker},\{\mathrm{T}\},\{\mathrm{X}\}, \mathrm{X}^{\prime}\right)\right)\right)==1$

$$
\text { z3 }=0 \text {; }
$$

else

$$
\mathrm{z} 3=\operatorname{diff}\left(\operatorname{subs}(\operatorname{ker},\{\mathrm{T}\},\{\mathrm{X}\}), \mathrm{X}^{\prime}\right) \text {; }
$$

end

ifisempty $(\operatorname{diff}($ ker, 'X' $))==1$

$$
\text { zz3 }=0 \text {; }
$$

else

$$
\text { zz3= diff(ker,' } \left.X^{\prime}\right) \text {; }
$$

end

$\mathrm{d} 2 \mathrm{u}(1)=\operatorname{subs}(z 2, \mathrm{a})+\operatorname{subs}(z z 3,\{X, T\},\{a, a\}) * u(1)+\operatorname{subs}(z 3, a)$

$* u(1)+\operatorname{subs}(k e r,\{X, T\},\{a, a\}) * d u(1)$;

ifisempty $(\operatorname{subs}(\operatorname{diff}(\operatorname{subs}(\operatorname{diff}(k e r, X), T, X), X), X, a))==1$; $\mathrm{s}=0$;

else

$\mathrm{s}=\operatorname{subs}(\operatorname{diff}(\operatorname{subs}(\operatorname{diff}($ ker,X), T, X), X), X, a);

end

ifisempty $(\operatorname{diff}(f, 3))==1$

$$
\mathrm{z} 4=0 \text {; }
$$

else

$\mathrm{z} 4=\operatorname{diff}(\mathrm{f}, 3)$;

end

ifisempty $\left(\operatorname{diff}\left(\operatorname{ker}, 2, \mathrm{X}^{\prime}\right)\right)==1$

$$
\mathrm{z} 5=0 \text {; }
$$

else z5= diff(ker,2,'X'); 
end

ifisempty(subs(diff(subs(ker, T, X), 2, X), X, a) )==1 $\mathrm{z} 6=0$;

else

$\mathrm{z} 6=\operatorname{subs}(\operatorname{diff}(\operatorname{subs}(\mathrm{ker}, \mathrm{T}, \mathrm{X}), 2, \mathrm{X}), \mathrm{X}, \mathrm{a})$;

end

ifisempty $(\operatorname{subs}(\operatorname{diff}(\operatorname{subs}($ ker, $T, X), X), X, a))==1$ $\mathrm{z} 7=0$;

else

$\mathrm{z} 7=\operatorname{subs}(\operatorname{diff}(\operatorname{subs}(\mathrm{ker}, \mathrm{T}, \mathrm{X}), \mathrm{X}), \mathrm{X}, \mathrm{a})$;

end

$\mathrm{d} 3 \mathrm{u}(1)=\operatorname{subs}(z 4, a)+\operatorname{subs}(\operatorname{subs}(z 5, \mathrm{~T}, \mathrm{X}), \mathrm{X}, \mathrm{a}) * \mathrm{u}(1)+\ldots$ $\mathrm{s}^{*} \mathrm{u}(1)+\operatorname{subs}(\operatorname{diff}(\operatorname{subs}(\operatorname{diff}(\mathrm{ker}, \mathrm{X}), \mathrm{T}, \mathrm{X}), \mathrm{X}, \mathrm{a})) * \operatorname{du}(1)+\ldots$ $\mathrm{z} 6 * \mathrm{u}(1)+2 * \mathrm{z} 7 * \mathrm{du}(1)+\operatorname{subs}(\operatorname{ker},\{\mathrm{X}, \mathrm{T}\},\{\mathrm{a}, \mathrm{a}\}) * \mathrm{~d} 2 \mathrm{u}(1)$;

ifisempty $(\operatorname{diff}(\mathrm{f}, 4))==1$

$\mathrm{z} 8=0$;

else

$\mathrm{z} 8=\operatorname{diff}(f, 4)$;

end

ifisempty $\left(\operatorname{diff}\left(\right.\right.$ ker, $\left.\left.3, \mathrm{X}^{\prime}\right)\right)==1$

$\mathrm{z} 9=0$;

else

$\mathrm{z} 9=\operatorname{diff}\left(\mathrm{ker}, 3,{ }^{\prime} \mathrm{X}\right)$;

end

ifisempty $(\operatorname{subs}(\operatorname{diff}(\operatorname{ker}, \mathrm{X}, 2), \mathrm{T}, \mathrm{X}))==1$ $\mathrm{z} 10=0$;

else

$\mathrm{z} 10=\operatorname{subs}(\operatorname{diff}(\mathrm{ker}, \mathrm{X}, 2), \mathrm{T}, \mathrm{X}) ;$

end

ifisempty $(\operatorname{subs}(\operatorname{diff}(z 10, X), X, a))==1$

z1010 $=0$;

else

$z 1010=\operatorname{subs}(\operatorname{diff}(z 10, X), X, a) ;$

end

ifisempty $\left(\operatorname{diff}\left(\mathrm{ker}, 2, \mathrm{X}^{\prime}\right)\right)==1$

$\mathrm{z} 11=0$;

else

$\mathrm{z} 11=\operatorname{diff}\left(\mathrm{ker}, 2,{ }^{\prime} \mathrm{X}\right)$;

end

ifisempty (subs(diff(ker,'X'),T,X))==1

$\mathrm{z} 12=0$;

else

z12=subs(diff(ker,'X'),T,X);

end

ifisempty $(\operatorname{subs}(\operatorname{diff}(\mathrm{z} 12, \mathrm{X}, 2), \mathrm{X}, \mathrm{a}))==1$

z1212 $=0$;

else

$z 1212=\operatorname{subs}(\operatorname{diff}(z 12, X, 2), X, a) ;$

end

ifisempty $(\operatorname{subs}(\operatorname{diff}(\operatorname{subs}($ ker, $T, X), X, 3), X, a))==1$ $\mathrm{z} 14=0$;

else

z14=subs(diff(subs(ker, T, X), X, 3), X, a);

end

ifisempty(subs(diff(subs(ker, T, X), X, 2), X, a))==1 $\mathrm{z} 15=0$;

else

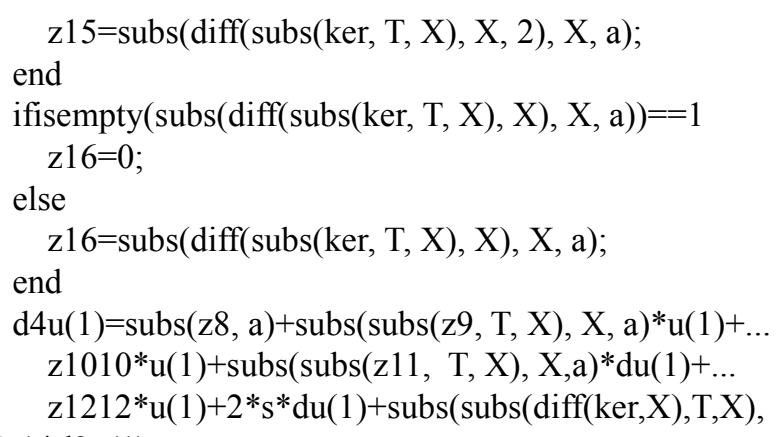

z15=subs(diff(subs(ker, T, X), X, 2), X, a);

end

ifisempty(subs(diff( $(\operatorname{subs}(\mathrm{ker}, \mathrm{T}, \mathrm{X}), \mathrm{X}), \mathrm{X}, \mathrm{a}))==1$ $\mathrm{z} 16=0$;

else

z16=subs(diff(subs(ker, T, X), X), X, a);

end

$\mathrm{d} 4 \mathrm{u}(1)=\operatorname{subs}(\mathrm{z} 8, \mathrm{a})+\operatorname{subs}(\operatorname{subs}(\mathrm{z} 9, \mathrm{~T}, \mathrm{X}), \mathrm{X}, \mathrm{a}) * \mathrm{u}(1)+\ldots$ $z 1010 * u(1)+\operatorname{subs}(\operatorname{subs}(z 11, T, X), X, a) * d u(1)+\ldots$

$\mathrm{z} 1212 * u(1)+2 * \mathrm{~s} * \mathrm{du}(1)+\operatorname{subs}(\operatorname{subs}(\operatorname{diff}(\mathrm{ker}, \mathrm{X}), \mathrm{T}, \mathrm{X})$,

$\mathrm{X}, \mathrm{a}) * \mathrm{~d} 2 \mathrm{u}(1)+\ldots$

$\mathrm{z} 14 * \mathrm{u}(1)+3 * \mathrm{z} 15 * \mathrm{u}(1)+3 * \mathrm{z} 16 * \mathrm{~d} 2 \mathrm{u}(1)+\operatorname{subs}(\operatorname{ker},\{\mathrm{X}, \mathrm{T}\},\{\mathrm{a}, \mathrm{a}\})$

$* \mathrm{~d} 3 \mathrm{u}(1)$;

ifisempty $(\operatorname{diff}(f, 5))==1$

$\mathrm{z} 17=0$;

else

$\mathrm{z} 17=\operatorname{diff}(\mathrm{f}, 5)$;

end

ifisempty $\left(\operatorname{diff}\left(\operatorname{ker}, 4,{ }^{\prime} X^{\prime}\right)\right)==1$

$\mathrm{z} 18=0$;

else

z18=diff(ker,4,'X');

end

ifisempty $(\operatorname{subs}(\operatorname{diff}(\operatorname{ker}, \mathrm{X}, 3), \mathrm{T}, \mathrm{X}))==1$

$\mathrm{z} 19=0$;

else

z19=subs(diff(ker,X,3),T, X);

end

ifisempty $(\operatorname{subs}(\operatorname{diff}(\mathrm{z} 19, \mathrm{X}), \mathrm{X}, \mathrm{a}))==1$

z1919=0;

else

z1919=subs(diff(z19,X), X, a);

end

ifisempty $\left(\operatorname{diff}\left(\right.\right.$ ker, $\left.\left.3, X^{\prime}\right)\right)==1$

$\mathrm{z} 20=0$;

else

z20=diff(ker,3,'X');

end

ifisempty $\left(\operatorname{subs}\left(\operatorname{diff}\left(\operatorname{ker}, 2,{ }^{\prime} X^{\prime}\right), T, X\right)\right)==1$

$\mathrm{z} 21=0$;

else

z21=subs(diff(ker,2,'X'),T,X );

end

ifisempty $(\operatorname{subs}(\operatorname{diff}(z 21, X, 2), X, a))==1$

z $2121=0$;

else

z2121 =subs(diff(z21,X,2),X,a);

end

ifisempty $(\operatorname{subs}(\operatorname{diff}(\mathrm{ker}, \mathrm{X}, 2), \mathrm{T}, \mathrm{X}))==1$

$\mathrm{z} 22=0$;

else

z22=subs(diff(ker, X,2),T, X);

end

ifisempty $(\operatorname{subs}(\operatorname{diff}(z 22, X), X, a))==1$ $\mathrm{z} 2222=0$;

else 
Elgaili Abdalla Elhassan Ibrahim et al: Comparison Between Adomain Decomposition Method and Numerical Solutions of Linear Volterra Integral Equations of the Second Kind by Using the Fifth Order of Non-Polynomial Spline Functions
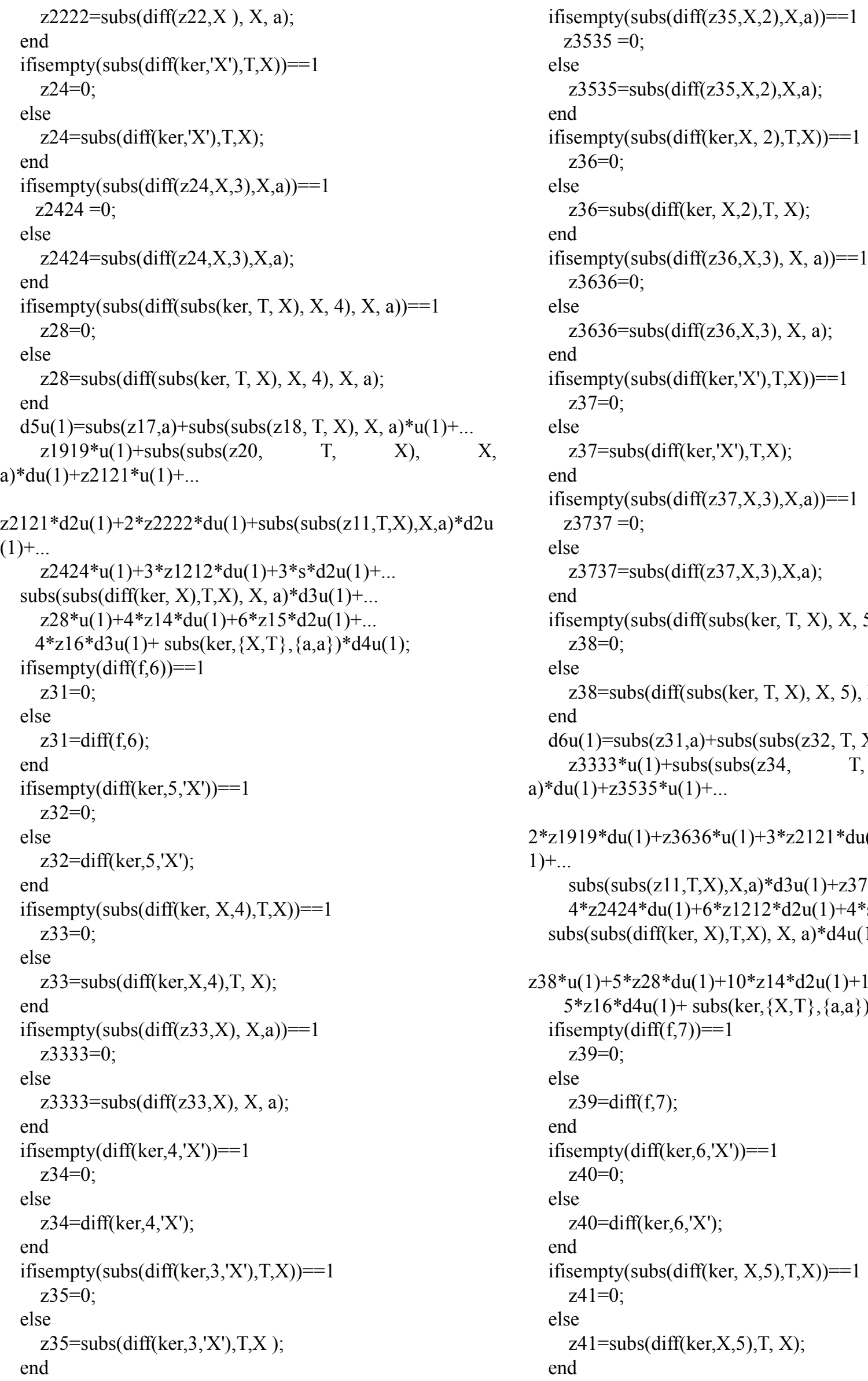

ifisempty(subs(diff( $\operatorname{subs}($ ker, T, X), X, 5), X, a ) $)==1$ $\mathrm{z} 38=0$;

else $z 38=\operatorname{subs}(\operatorname{diff}(\operatorname{subs}(k e r, T, X), X, 5), X, a) ;$ end $\mathrm{d} 6 \mathrm{u}(1)=\operatorname{subs}(z 31, a)+\operatorname{subs}(\operatorname{subs}(z 32, T, X), X, a) * u(1)+\ldots$ $z 3333 * u(1)+\operatorname{subs}(\operatorname{subs}(z 34, \quad T, \quad X), \quad X$, a) $* d u(1)+z 3535 * u(1)+\ldots$

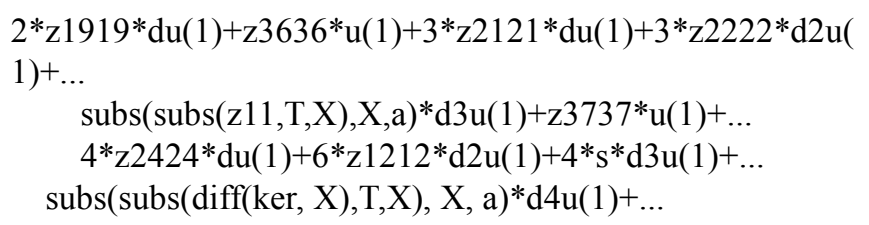


ifisempty $(\operatorname{subs}(\operatorname{diff}(z 41, X), X, a))==1$

$$
\text { z4141=0; }
$$

else

$$
\text { z4141=subs(diff(z41,X), X, a); }
$$

end

ifisempty $\left(\operatorname{diff}\left(\operatorname{ker}, 5, \mathrm{X}^{\prime}\right)\right)==1$

$$
\mathrm{z} 42=0 \text {; }
$$

else

$\mathrm{z} 42=\operatorname{diff}\left(\mathrm{ker}, 5, \mathrm{X}^{\prime}\right)$;

end

ifisempty $\left(\operatorname{subs}\left(\operatorname{diff}\left(\mathrm{ker}, 4, \mathrm{X}^{\prime}\right), \mathrm{T}, \mathrm{X}\right)\right)==1$

$$
\mathrm{z} 43=0 \text {; }
$$

else

$$
\mathrm{z} 43=\operatorname{subs}\left(\operatorname{diff}\left(\mathrm{ker}, 4, \mathrm{X}^{\prime}\right), \mathrm{T}, \mathrm{X}\right) \text {; }
$$

end

ifisempty $(\operatorname{subs}(\operatorname{diff}(z 43, X, 2), X, a))==1$

z4343 =0;

else

$z 4343=\operatorname{subs}(\operatorname{diff}(z 43, X, 2), X, a) ;$

end

ifisempty $(\operatorname{subs}(\operatorname{diff}(\operatorname{ker}, \mathrm{X}, 3), \mathrm{T}, \mathrm{X}))==1$

$$
\text { z44=0; }
$$

else

$\mathrm{z} 44=\operatorname{subs}(\operatorname{diff}(\mathrm{ker}, \mathrm{X}, 3), \mathrm{T}, \mathrm{X})$;

end

ifisempty $(\operatorname{subs}(\operatorname{diff}(z 44, X, 3), X, a))==1$ $\mathrm{z} 4444=0$;

else

$z 4444=\operatorname{subs}(\operatorname{diff}(z 44, X, 3), X, a) ;$

end

ifisempty $(\operatorname{subs}(\operatorname{diff}($ ker,X, 2), T,X) $)==1$

$$
\mathrm{z} 45=0 \text {; }
$$

else

$\mathrm{z} 45=\operatorname{subs}(\operatorname{diff}($ ker, $X, 2), \mathrm{T}, \mathrm{X})$;

end

ifisempty $(\operatorname{subs}(\operatorname{diff}(z 45, X, 3), X, a))==1$

$$
\mathrm{z} 4545=0 \text {; }
$$

else

$z 4545=\operatorname{subs}(\operatorname{diff}(z 45, X, 3), X, a) ;$

end

ifisempty $\left(\operatorname{subs}\left(\operatorname{diff}\left(\mathrm{ker}, \mathrm{X}^{\prime}\right), \mathrm{T}, \mathrm{X}\right)\right)==1$

$\mathrm{z} 46=0$;

else

$\mathrm{z} 46=\operatorname{subs}\left(\operatorname{diff}\left(\mathrm{ker}, \mathrm{X}^{\prime}\right), \mathrm{T}, \mathrm{X}\right)$;

end

ifisempty $(\operatorname{subs}(\operatorname{diff}(z 46, X, 5), X, a))==1$

$\mathrm{z} 4646=0$;

else

$z 4646=\operatorname{subs}(\operatorname{diff}(z 46, X, 5), X, a) ;$

end

ifisempty $(\operatorname{subs}(\operatorname{diff}(\operatorname{subs}(\operatorname{ker}, T, X), X, 6), X, a))==1$ $\mathrm{z} 47=0$;

else

$\mathrm{z} 47=\operatorname{subs}(\operatorname{diff}(\operatorname{subs}($ ker, $T, X), X, 6), X, a) ;$

end

$\mathrm{d} 7 \mathrm{u}(1)=\operatorname{subs}(z 39, a)+\operatorname{subs}(\operatorname{subs}(z 40, T, X), X, a) * u(1)+\ldots$ $\mathrm{z} 4141 * u(1)+\operatorname{subs}(\operatorname{subs}(\mathrm{z} 42$, a)*du(1)+z4343*u(1)+...

$2 * z 3333 * d u(1)+\operatorname{subs}(\operatorname{subs}(z 34, T, X), X, a) * d 2 u(1)+\ldots$

$z 4444 * u(1)+3 * z 3535 * d u(1)+3 * z 1919 * d 2 u(1)+\ldots$

$\mathrm{z} 4545 * \mathrm{u}(1)+4 * \mathrm{z} 3636 * \mathrm{du}(1)+6 * \mathrm{z} 2121 * \mathrm{~d} 2 \mathrm{u}(1)+\ldots$

$4 * z 2222 * d 3 u(1)+\operatorname{subs}(\operatorname{subs}(z 20, T, X), X, a) * d 3 u(1)+\ldots$

$\operatorname{subs}(\operatorname{subs}(z 11, T, X), X, a) * d 4 u(1)+z 4646 * u(1)+5 * z 3737 * d u(1$

)$+\ldots$

$10 * \mathrm{z} 2424 * \mathrm{~d} 2 \mathrm{u}(1)+10 * \mathrm{z} 1212 * \mathrm{~d} 3 \mathrm{u}(1)+5 * \mathrm{~s} * \mathrm{~d} 4 \mathrm{u}(1)+\ldots$

$\operatorname{subs}(\operatorname{subs}(\operatorname{diff}(k e r, X), T, X), X, a) * d 5 u(1)+\ldots$

$z 47 * u(1)+6 * z 38 * d u(1)+15 * z 28 * d 2 u(1)+20 * z 14 * d 3 u(1)+\ldots$

$15 * \mathrm{z} 15 * \mathrm{~d} 4 \mathrm{u}(1)+6 * \mathrm{z} 16 * \mathrm{~d} 5 \mathrm{u}(1)+\operatorname{subs}(\mathrm{ker},\{\mathrm{X}, \mathrm{T}\},\{\mathrm{a}, \mathrm{a}\}) * \mathrm{~d} 6 \mathrm{u}(1) ;$

$\mathrm{a}(1)=-\mathrm{d} 6 \mathrm{u}(1)$;

$\mathrm{b}(1)=\mathrm{d} 7 \mathrm{u}(1)$;

$\mathrm{c}(1)=\mathrm{du}(1)-\mathrm{d} 7 \mathrm{u}(1)$

$\mathrm{d}(1)=(1 / 2) *(\mathrm{~d} 2 \mathrm{u}(1)-\mathrm{d} 6 \mathrm{u}(1))$;

$\mathrm{e}(1)=(1 / 6) *(\mathrm{~d} 3 \mathrm{u}(1)+\mathrm{d} 7 \mathrm{u}(1))$;

$\mathrm{r}(1)=(1 / 24) *(\mathrm{~d} 4 \mathrm{u}(1)+\mathrm{d} 6 \mathrm{u}(1))$;

$\mathrm{z}(1)=(1 / 120) *(\mathrm{~d} 5 \mathrm{u}(1)-\mathrm{d} 7 \mathrm{u}(1))$;

$\mathrm{g}(1)=\mathrm{u}(1)+\mathrm{d} 6 \mathrm{u}(1)$;

for $\mathrm{i}=1: \mathrm{n}-1$

$\mathrm{u}(\mathrm{i}+1)=\mathrm{a}(\mathrm{i}) * \cos (\mathrm{h})+\mathrm{b}(\mathrm{i}) * \sin (\mathrm{h})+\mathrm{h}^{*} \mathrm{c}(\mathrm{i})+\mathrm{d}(\mathrm{i}) * \mathrm{~h}^{\wedge} 2+$

e(i) $* h^{\wedge} 3+r(i) * h^{\wedge} 4+z(i) * h^{\wedge} 5+g(i)$;

$\mathrm{du}(\mathrm{i}+1)=-\mathrm{a}(\mathrm{i}) * \sin (\mathrm{h})+\mathrm{b}(\mathrm{i}) * \cos (\mathrm{h})+\mathrm{c}(\mathrm{i})+2 * \mathrm{~d}(\mathrm{i}) * \mathrm{~h}+$

$3 * e(i) * h^{\wedge} 2+4 * r(i) * h^{\wedge} 3+5 * z(i) * h^{\wedge} 4$;

$\mathrm{d} 2 \mathrm{u}(\mathrm{i}+1)=-\mathrm{a}(\mathrm{i}) * \cos (\mathrm{h})-\mathrm{b}(\mathrm{i}) * \sin (\mathrm{h})+2 * \mathrm{~d}(\mathrm{i})+6 * \mathrm{e}(\mathrm{i}) * \mathrm{~h}$

$+12 * r(i) * h^{\wedge} 2+20 * z(i) * h^{\wedge} 3$;

$\mathrm{d} 3 \mathrm{u}(\mathrm{i}+1)=\mathrm{a}(\mathrm{i}) * \sin (\mathrm{h})-\mathrm{b}(\mathrm{i}) * \cos (\mathrm{h})+6 * \mathrm{e}(\mathrm{i})+24 * \mathrm{r}(\mathrm{i}) * \mathrm{~h}$

$+60 * z(i) * h^{\wedge} 2$

$\mathrm{d} 4 \mathrm{u}(\mathrm{i}+1)=\mathrm{a}(\mathrm{i}) * \cos (\mathrm{h})+\mathrm{b}(\mathrm{i}) * \sin (\mathrm{h})+24 * \mathrm{r}(\mathrm{i})+$

$120 * \mathrm{z}(\mathrm{i}) * \mathrm{~h}$;

$\mathrm{d} 5 \mathrm{u}(\mathrm{i}+1)=-\mathrm{a}(\mathrm{i}) * \sin (\mathrm{h})+\mathrm{b}(\mathrm{i}) * \cos (\mathrm{h})+120 * \mathrm{z}(\mathrm{i})$;

d6u(i+1) $=-a(i) * \cos (h)-b(i) * \sin (h)$;

$\mathrm{d} 7 \mathrm{u}(\mathrm{i}+1)=\mathrm{a}(\mathrm{i}) * \sin (\mathrm{h})-\mathrm{b}(\mathrm{i}) * \cos (\mathrm{h})$;

$a(i+1)=-d 6 u(i+1)$;

$b(i+1)=d 7 u(i+1)$;

$\mathrm{c}(\mathrm{i}+1)=\mathrm{du}(\mathrm{i}+1)-\mathrm{d} 7 \mathrm{u}(\mathrm{i}+1)$;

$\mathrm{d}(\mathrm{i}+1)=(1 / 2)^{*}(\mathrm{~d} 2 \mathrm{u}(\mathrm{i}+1)-\mathrm{d} 6 \mathrm{u}(\mathrm{i}+1))$;

$\mathrm{e}(\mathrm{i}+1)=(1 / 6) *(\mathrm{~d} 3 \mathrm{u}(\mathrm{i}+1)+\mathrm{d} 7 \mathrm{u}(\mathrm{i}+1))$;

$r(i+1)=(1 / 24) *(d 4 u(i+1)+d 6 u(i+1))$;

$\mathrm{z}(\mathrm{i}+1)=(1 / 120) *(\mathrm{~d} 5 \mathrm{u}(\mathrm{i}+1)-\mathrm{d} 7 \mathrm{u}(\mathrm{i}+1))$;

$\mathrm{g}(\mathrm{i}+1)=\mathrm{u}(\mathrm{i}+1)+\mathrm{d} 6 \mathrm{u}(\mathrm{i}+1)$;

end

err $=$ zeros $(1, n)$;

$\operatorname{disp}(\mathrm{h})$

disp('length of $x^{\prime}$ )

$\operatorname{disp}($ length $(\mathrm{x}))$

disp('length of $y^{\prime}$ ')

$\operatorname{disp}($ length $(y))$

for $\mathrm{k}=1: \mathrm{n}$

$\operatorname{err}(\mathrm{k})=\operatorname{abs}(\mathrm{u}(\mathrm{k})-\mathrm{y}(\mathrm{k}))$;

end

format long

$\begin{array}{llllll}\operatorname{disp}(' & \mathrm{i} & \mathrm{X} & \mathrm{Y} & \mathrm{u} & \text { err') }\end{array}$

[1:n; x; y; u; err]' 
figure(1)

plot(x, y, 'b --', 'linewidth', 3)

hold on

plot(x, u, 'r.-', 'linewidth', 1)

grid on

xlabel('X')

ylabel('Y')

title('exact vs. approx.')

legend('exact', 'approx.')

\section{Numerical Examples and Figures}

Example (2): Consider the VIE of second kind [10]:

$$
u(x)=x+\int_{0}^{x}(t-x) u(t) d t 0 \leq x \leq 1
$$

Where $f(x)=x$ and kernel $=t-x$, with $y=\sin x$

Table 1 present a comparison between the exact and numerical solution using linear [13] and fifth order nonpolynomial spline functions, where $u_{i}(x)$ denotes the approximate solution using non- polynomial spline functions, with $h=0.1$.

Table 1. Analytical and numerical solution of test example(2).

\begin{tabular}{llll}
\hline $\boldsymbol{x}$ & \multirow{2}{*}{ Exact solution } & $\boldsymbol{u}_{\boldsymbol{i}}(\boldsymbol{x})$ & \\
\cline { 3 - 4 } & & linear & Five order \\
\hline 0 & 0.00000000000000 & 0.00000000000000 & 0.00000000000000 \\
0.1 & 0.09983341664683 & 0.09983341664683 & 0.09983341668651 \\
0.2 & 0.19866933079506 & 0.19916808204436 & 0.19866933583213 \\
0.3 & 0.29552020666134 & 0.29800648787140 & 0.29552028832715 \\
0.4 & 0.38941834230865 & 0.39635111335875 & 0.38941890981745 \\
0.5 & 0.47942553860420 & 0.49420442535136 & 0.47942806425312 \\
0.6 & 0.56464247339504 & 0.59156887837027 & 0.56465102008993 \\
0.7 & 0.64421768723769 & 0.68844691467410 & 0.64424167375464 \\
0.8 & 0.71735609089952 & 0.78484096432036 & 0.71741482576318 \\
0.9 & 0.78332690962748 & 0.88075344522641 & 0.78345650410261 \\
1 & 0.84147098480790 & 0.97618676323008 & 0.84173434026604 \\
\hline
\end{tabular}

Table 2: present a comparison between the error in our methods and other method] where error=|exact value numerical

Table 2. Comparison between the error with

\begin{tabular}{lll}
\hline $\boldsymbol{x}$ & Error in linear & Error in five order \\
\hline 0 & 0.00000000000000 & 0.00000000000000 \\
0.1 & 0.00000000000000 & 0.00000000003968 \\
0.2 & 0.00049875124930 & 0.00000000503707 \\
0.3 & 0.00248628121006 & 0.00000008166582 \\
0.4 & 0.00693277105010 & 0.00000056750880 \\
0.5 & 0.01477888674716 & 0.00000252564892 \\
0.6 & 0.02692640497523 & 0.00000854669489 \\
0.7 & 0.04422922743640 & 0.00002398651695 \\
0.8 & 0.06748487342084 & 0.00005873486365 \\
0.9 & 0.09742653559892 & 0.00012959447513 \\
1 & 0.13471577842218 & 0.00026335545814 \\
\hline
\end{tabular}

Example (3): Consider the VIE of second kind [14]:

$$
u(x)=1+\int_{0}^{x}(t-x) u(t) d t 0 \leq x \leq 1
$$

Where $f(x)=1$ and kernel $=t-x$, with $y=\cos x$

Table 3 present a comparison between the exact and numerical solution using linear [13] and five order nonpolynomial spline functions, where $u_{i}(x)$ denotes the approximate solution using non- polynomial spline functions, with $h=0.1$.

Table 3. Exact and numerical solution of test example(3).

\begin{tabular}{lcll}
\hline $\boldsymbol{x}$ & \multirow{2}{*}{ Exact solution } & $\boldsymbol{u}_{\boldsymbol{i}}(\boldsymbol{x})$ & \\
\cline { 3 - 4 } & & linear & Five order \\
\hline 0 & 1.00000000000000 & 1.00000000000000 & 1.00000000000000 \\
0.1 & 0.99500416527803 & 0.99500416527803 & 0.99500416527803 \\
0.2 & 0.98006657784124 & 0.99003328892062 & 0.98006657783728 \\
0.3 & 0.95533648912561 & 0.98508724623992 & 0.95533648861880 \\
0.4 & 0.92106099400289 & 0.98016591317098 & 0.92106098534563 \\
0.5 & 0.87758256189037 & 0.97526916626867 & 0.87758249662003 \\
0.6 & 0.82533561490968 & 0.97039688270455 & 0.82533529782125 \\
0.7 & 0.76484218728449 & 0.96554894026384 & 0.76484101853443 \\
0.8 & 0.69670670934717 & 0.96072521734230 & 0.69670315178005 \\
0.9 & 0.62160996827066 & 0.95592559294323 & 0.62160056477445 \\
1 & 0.54030230586814 & 0.95114994667438 & 0.54028001149101 \\
\hline
\end{tabular}

Table 4: present a comparison between the error in our methods and other method in [10] where error=|exact value numerical

Table 4. Comparison between the error with.

\begin{tabular}{lll}
\hline $\boldsymbol{x}$ & Error in linear & Error in five order \\
\hline 0 & 0.00000000000000 & 0.00000000000000 \\
0.1 & 0.00000000000000 & 0.00000000000000 \\
0.2 & 0.00996671107938 & 0.00000000000396 \\
0.3 & 0.02975075711431 & 0.00000000050681 \\
0.4 & 0.05910491916810 & 0.00000000865725 \\
0.5 & 0.09768660437829 & 0.00000006527035 \\
0.6 & 0.14506126779487 & 0.00000031708843 \\
0.7 & 0.20070675297935 & 0.00000116875006 \\
0.8 & 0.26401850799514 & 0.00000355756712 \\
0.9 & 0.33431562467256 & 0.00000940349622 \\
1 & 0.41084764080624 & 0.00002229437713 \\
\hline
\end{tabular}

The Figures show the comparison between the analytical and the approximate solution using linear and fifth order nonpolynomial spline functions for the examples.

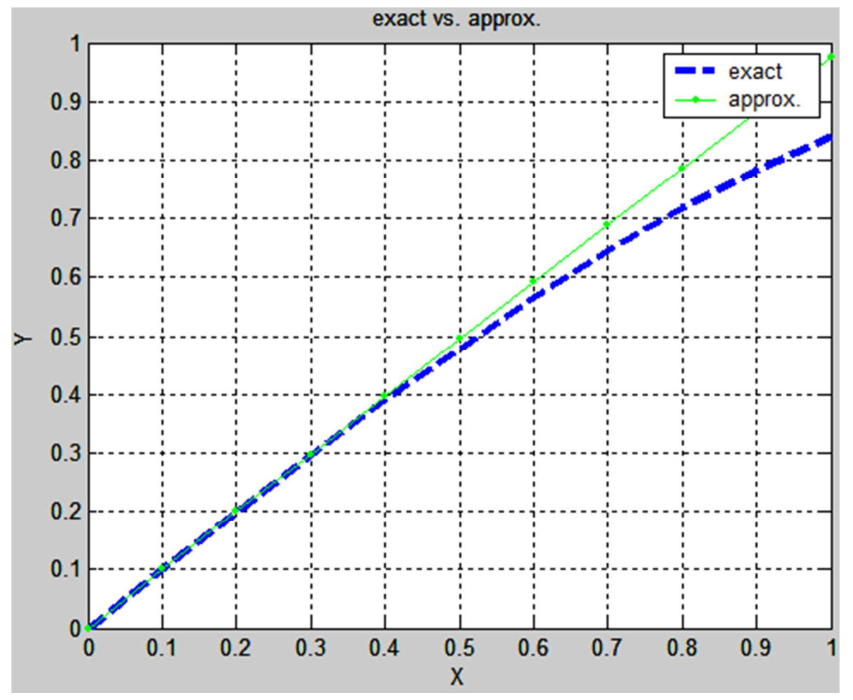

Figure 1. Comparison between the analytical and the approximate solution using linear non-polynomial spline functions for example (2). 


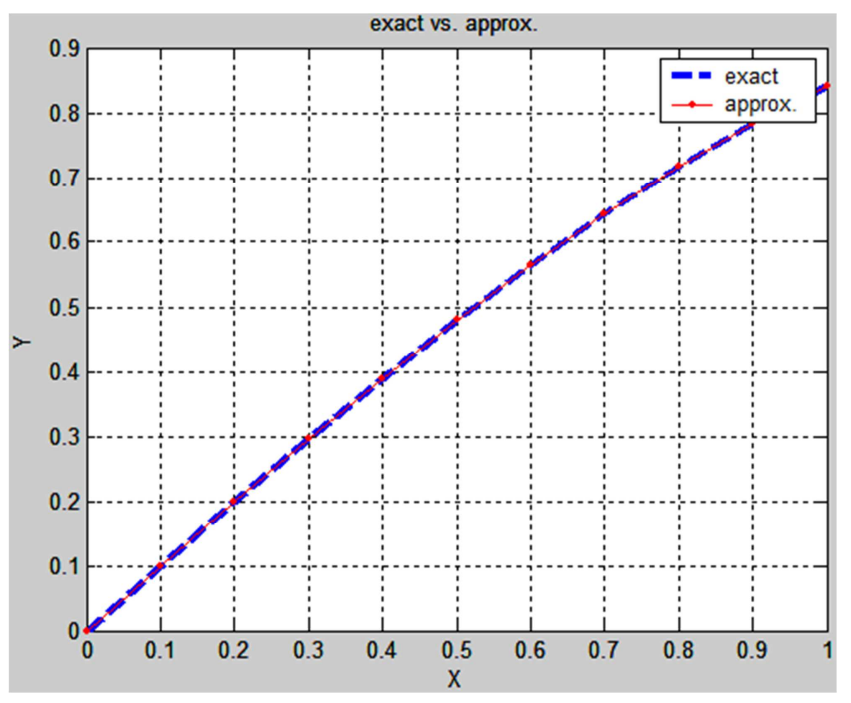

Figure 2. Comparison between the analytical and the approximate solution using fifth order non-polynomial spline functions for example (2).

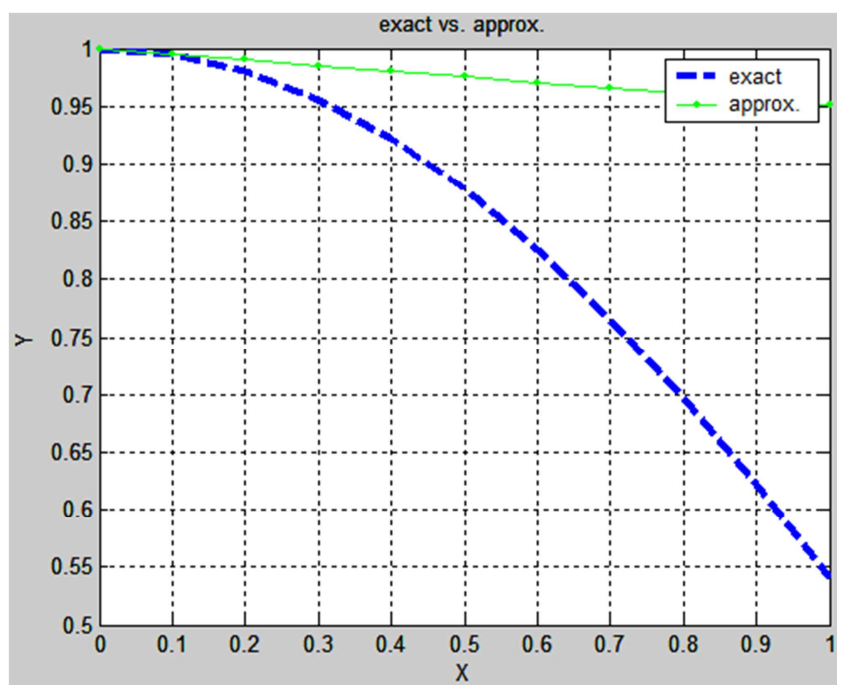

Figure 3. Comparison between the analytical and the approximate solution using linear non-polynomial spline functions for example (3).

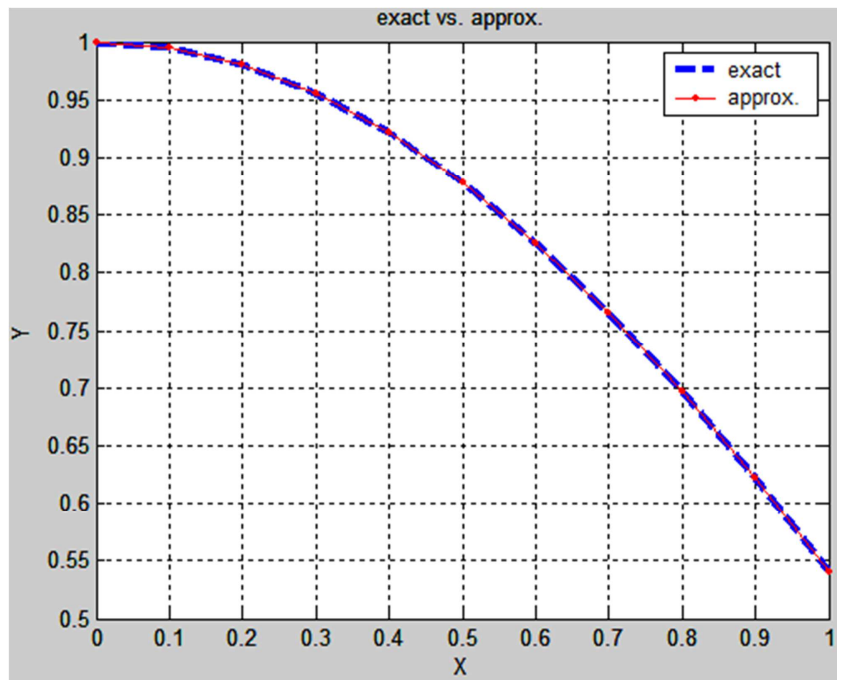

Figure 4. Comparison between the analytical and the approximate solution using fifth order non-polynomial spline functions for example (3).

\section{Results}

In general the methods which are used in this paper proved their effectiveness in solve linear Volterra Integral Equations of second kind numerically and finding an accurate results. The Figures show a comparison between the Adomain Decomposition and numerical solution which was presented in example (2) and example (3), the results show that the fifth order non-polynomial Spline functions gives the best approximation than the linear non-polynomial Spline functions.

\section{Conclusion}

Finally we found that the fifth order non- polynomial Spline functions characterized with an easy, fast and accuracy is high comparing with the less fifth order nonpolynomial Spline functions and the results that we obtained show that the fifth order non-polynomial Spline functions gives the best approximation to solve the linear nonpolynomial Spline functions. The proposed scheme is simple and computationally attractive and its accuracy is high and we can simply execute this method by Matlab.

\section{References}

[1] Abdel Radi. A. A and Samia. A. Y, Comparison Between Analysis Solutions of Volterra and Fredholm Integral Equations of Second Kind, EPH - International Journal of Mathematics and Statistics vol. 6, issue 9, p.p 3-5, 2020.

[2] Haq, F. I. (2009). Numerical Solution of Bounded Value Problem and Initial Value Problems Using Spline Function. ph.d, thesis, GhulamIshaq Institute of Engineering Science and Technology, Pakistan.

[3] HARBI, S, MURAD, M and MAJEED, M 2015-A solution of second integral equation using third order Non-polynomial Spline function. College of Science for Women Baghdad University.

[4] H. Brunner, theory and numerical solution of Volterra functional integral equations, Hong Kong Baptist University, (2010).

[5] HOSSINPOUR, A 2012- The Solve of Integral Differential Equation by Non-polynomial Spline Function and Quadrature Formula. International Conference on Applied Mathematics and Pharmaceutical science Jan, pp 7-8, pp. 595-597.

[6] LIMA, P and DIAGO, T 1997- An extrapolation method for a Volterra integral equation with weakly singular kernel Appl. Numer. Math, vol. 24, pp. 131-148.

[7] Najwa, S and Mohammed, S presented a numerical solution for linear Voltera integral equations with weakly singular kernel by using non-polynomial spline function from the fifth degree, pp. 109-110.

[8] Majeed. S. N (2014) Solution Of Second Kind Volterra Integro Equation Using Linear Non Polynomial Spline Equation. Mathematical Theory and Modeling. 
[9] M. S. Islam, M. Z. I. Bangalee, A. K.. Khan, and, A, Halder," approximate solution of systems of VIE's of second kind by Adomain decomposition method," Dhaka university journal of science, vol. 63, no. 1, pp. 15-18, 2007.

[10] Rahman, M. M, Hakim M. A., Hassan M. K., Alam M. K. and Nowsher, L., 2012, Numerical Solution of Volterra Integral Equations of Second kind with the Help of Chebyshev Polynomials, Annals of Pure and Applied Mathematics, 1 (2): 158-167.

[11] Ramadan, M. A.; EL-Danaf, T.; and E. I. Abdaal, F (2007). Application of the Non- Polynomial Spline Approach to the Solution of the Burgers Equation. The Open Applied Mathematics Journal (1): 15-20.

[12] Rice, J. R. (1985). Numerical Method software and Analysis. Software and analysis, Mcgra Hill.

[13] Sara. H. H, (2013), Algorithms for Solving Volterra Integral
Equations Using Non-Polynomial Notch Functions, College of Science for Women Baghdad University.

[14] Tahmasbi, A. (2008). New Approach numerical solution of Linear Volterra Integral Equations of Second kind. 3 (32), 1607-1610.

[15] Taqi, A; Jumaa, B (2016). Non- polynomial spline functions to solve a system of two non- linear volterra integral equations. Kirkuk University, Scientific Studies.

[16] WAZWAZ, A 2011-Linear and Non Linear Integral Equation Method and Application. Higher Education Press, Beijing, p. 66-69.

[17] Zarebnia, M.; Hoshyar, M.; and Sedahti, M. (2011). NonPolynomial Spline Method for the Solution of Problem in Calculus of Variations. word Academy Engendering Technology (51): 986-991. 